\title{
Associação da ansiedade traço com sintomas de transtornos alimentares em bailarinos
}

http://dx.doi.org/10.11606/1807-5509201900020293

\author{
Nycolle Martins REIS* \\ Andreia PELEGRINI* \\ Leonessa BOING* \\ Jéssica MORATELLI* \\ Adriana Coutinho de Azevedo GUIMARÃES* \\ *Departamento de \\ Educação Física, \\ Centro de Ciências da \\ Saúde e do Esporte, \\ Universidade do \\ Estado de Santa \\ Catarina, Florianópolis, \\ SC, Brasil.
}

\section{Resumo}

Bailarinos sofrem exigências em função de um corpo magro, o que pode gerar ansiedade e deixá-los por vezes mais propensos aos sintomas transtornos alimentares. Sendo assim, esse estudo objetivou analisar a associação da ansiedade traço com sintomas de transtornos alimentares controlados por nível socioeconômico, grau de escolaridade e modalidade em bailarinos do $31^{\circ}$ Festival de Dança de Joinville na cidade de Joinville/Santa Catarina (SC). Participaram do estudo 156 bailarinos do 31 ${ }^{\circ}$ Festival de Dança de Joinville - Santa Catarina, os quais responderam a um questionário autoaplicável contendo as variáveis sociodemográficas, medidas antropométricas, Inventário de Ansiedade Traço-Estado (IDATE), Teste de Atitudes Alimentares (EAT-26) e Bulimic Inventory Test Edinburgh (BITE). Observou-se prevalência elevada de ansiedade traço (82\%). Houve associação entre sintomas de bulimia nervosa com a ansiedade traço, apontando que os bailarinos com sintomas de bulimia apresentaram probabilidade maior 1,15 (IC95\% = 1,05-1,27) em ter ansiedade elevada e aqueles bailarinos com gravidade de sintomas de bulimia apresentaram uma probabilidade maior de 1,13 $(I C 95 \%=1,01-1,28)$ em ter ansiedade traço elevada. Encontrou-se grande prevalência e forte associação da ansiedade traço com os transtornos da alimentação de bailarinos durante o Festival de Dança de Joinville.

Palavras-chave: Ansiedade; Bulimia Nervosa; Anorexia Nervosa; Dança.

\section{Introdução}

Ao longo dos tempos a dança passou por transformaçóes e deixou de ser vista apenas como uma manifestação religiosa/ritual ${ }^{1,2}$ e/ou lúdica, para ser vista também como artística, performática ${ }^{3}$. Neste sentido, a dança promove representaçóes de sentimentos humanos por meio de expressóes corporais ritmadas ${ }^{1}$. Além disso, ela engloba técnicas corporais e pressóes psicológicas antes apenas comentadas em atletas de alto rendimento ${ }^{3}$.

Em função do grande numero de apresentações e/ ou competições e das exigências da própria dança, os bailarinos podem apresentar um aumento substancial na ansiedade ${ }^{4}$. A ansiedade, neste caso, pode ser definida como uma resposta ao estresse, na qual o indivíduo entra em um estado de nervosismo por um possível perigo eminente no qual desconhece a causa ${ }^{2}$. No caso, a ansiedade-traço é a pré-disposição que um indivíduo apresenta ao longo da vida para demonstrar os sentimentos de ansiedade ${ }^{3}$.

Bailarinos estâo frequentemente rodeados de sentimentos que podem gerar ansiedade, especialmente, quando em situaçóes competitivas, o que pode afetar seu desempenho técnico ${ }^{4}$. No contexto da dança há comentários de colegas e coreógrafos sobre o corpo do bailarino, reforçando o desejo pelo corpo magro já implícito na modalidade, deixando estes por vezes mais propensos aos transtornos alimentares 5 .

Os transtornos alimentares são recorrentes e comuns em bailarinos, como mostra os valores identificados em uma metanálise em que bailarinos têm três vezes mais chance de apresentar transtornos alimentares que indivíduos que não dançam ${ }^{6}$. E, devido a todo contexto de pressóes sobre o corpo aos quais sofrem estes bailarinos, as 
competiçôes são ambientes que permeiam sentimentos de ansiedade, os quais estão atrelados aos transtornos alimentares, uma vez que os bailarinos mais neuróticos com o próprio corpo, são mais ansiosos e apresentam mais sintomas de transtornos alimentares ${ }^{7-9}$.

\section{Método}

Estudo transversal composto por 157 bailarinos de ambos os sexos das modalidades Balé Clássico $(\mathrm{n}=48)$, Dança Contemporânea $(\mathrm{n}=23)$ e Danças Urbanas $(\mathrm{n}=86)$, inscritos na categoria avançada (nascidos no ano de 1996, acima de 16 anos), subgênero conjunto, do $31^{\circ}$ Festival de Dança de Joinville na cidade de Joinville/SC, selecionados por conveniência ${ }^{10}$. O Festival de Dança de Joinville/SC é conceituado como o maior festival de dança do mundo, pelo Guinness $B o o k^{11}$, recebendo bailarinos do Brasil e de países da América Latina ${ }^{10}$.

Este projeto foi aprovado pelo Comitê de Ética em Pesquisa com Seres Humanos (CEPSH) da Universidade do Estado de Santa Catarina (UDESC), parecer $\mathrm{n}^{\circ}$ 200.140. Foram considerados elegíveis os bailarinos que se propuseram a participar voluntariamente do estudo e que assinaram o Termo de Consentimento Livre e Esclarecido (TCLE) ${ }^{10}$. Os grupos de dança foram contatados pelas Pesquisadoras por e-mails, telefones e sites de relacionamentos (facebook; twitter) após divulgação de selecionados pelo festival, a fim de verificar o interesse em participar da pesquisa, e realizar um levantamento do número de bailarinos por grupo e estratégia de coleta de dados ${ }^{10}$. Sendo assim, encaminhou-se por e-mail aos pais e/ou responsáveis de bailarinos menores de 18 anos o TCLE, os quais foram entregues posteriormente assinados pelos pais e/ou responsáveis no dia da coleta ${ }^{10}$.

A coleta de dados foi realizada durante o mês de julho de 2013, antes das apresentaçóes da mostra competitiva das respectivas modalidades de dança. Os bailarinos responderam de forma presencial a um questionário autoaplicável, sendo auxiliados pelas pesquisadoras quando necessário, e tiveram garantia de sigilo tanto das respostas como de suas identidades. Por fim, os participantes receberam esclarecimento quanto ao correto preenchimento do questionário e foram elucidados sobre seus objetivos ${ }^{10}$. Este questionário foi dividido em três blocos:

O primeiro bloco com informaçóes gerais contidas de forma objetiva no questionário - idade (em anos
Neste sentido, o presente estudo teve como objetivo analisar a associaçáo da ansiedade traço com sintomas de transtornos alimentares controlados por nível socioeconômico, grau de escolaridade e modalidade em bailarinos do $31^{\circ}$ Festival de Dança de Joinville na cidade de Joinville/Santa Catarina (SC).

completos), sexo, escolaridade, estado conjugal (com ou sem companheiro), ocupação funcional, presença de doença (sim; não) e o nível econômico e variáveis antropométricas (peso corporal e estatura). O nível econômico foi avaliado por meio do instrumento denominado Critério de Classificação Econômica Brasil (CCEB) proposto pela Associaçấo Brasileira de Empresas de Pesquisa ${ }^{12}$, que classifica os indivíduos em estratos (A1, A2, B1, B2, C1, C2, D, E), a partir da soma dos pontos de cada questão. Para fins estatísticos as variáveis foram agrupadas em: baixo $(\mathrm{C} 1+\mathrm{C} 2+\mathrm{D}+\mathrm{E})$; médio $(\mathrm{B} 1+\mathrm{B} 2)$ e alto $(\mathrm{A} 1+\mathrm{A} 2)$. $O$ peso corporal e a estatura foram coletadas por meio de medidas autorreferidas. Por tratar-se de adultos em sua maioria, a correspondência entre medidas referidas e aferidas é satisfatória, de modo que o uso deste método tem sido considerado uma alternativa viável em situaçôes em que não é possível a obtenção direta dessas informaçóes ${ }^{13}$, ou quando e preciso tornar o estudo mais rápido e acessível quanto aos custos ${ }^{14}$. Por meio das medidas referidas de peso corporal e estatura fez-se o cálculo para o índice de massa corporal (IMC).

O segundo bloco apresenta o Inventário de Ansiedade Traço-Estado (IDATE) que é um dos instrumentos mais utilizados para verificar a ansiedade, o qual foi desenvolvido por SPIELbERger et al. ${ }^{15}$. Foi validado para populaçáo brasileira ${ }^{16}$, e utilizado em outro estudo com bailarinas ${ }^{4}$. O IDATE apresenta uma escala que avalia a ansiedade enquanto estado (IDATE-E) e outra que acessa a ansiedade enquanto traço (IDATE-T). Para a quantificação e interpretação das respostas, atribuiu-se a pontuação correspondente a resposta dada para cada uma das perguntas. Os escores para as perguntas de caráter positivo são invertidos, ou seja, se o indivíduo responder 4, atribui-se valor 1 na codificação; se responder 3, atribui-se valor 2 ; se responder 2 , atribui-se valor 3 ; e se responder 1, atribui-se valor 4. Para o IDATEtraço, as perguntas negativas são: $2,3,4,5,8,9,11$, $12,14,15,17,18,20$; e as positivas: $1,6,7,10,13$, 16, 19. Para efeito deste estudo optou-se pelo uso 
apenas da ansiedade traço pois esta se refere à prédisposição que um indivíduo apresenta ao longo da vida para demonstrar os sentimentos de ansiedade ${ }^{3}$, dessa maneira, como o mesmo reage frente a situaçóes consideradas pessoalmente como ameaçadoras; o que pode ser observado em situações de competições como a abordada neste estudo. $\mathrm{O}$ escore de cada parte varia de 20 a 80 pontos, sendo que, os escores podem indicar um baixo grau de ansiedade (0-49) e um grau elevado de ansiedade (maior ou igual a 50$)^{4}$.

O terceiro bloco foi composto de dois instrumentos e fez avaliação dos sintomas de transtornos alimentares. $\mathrm{O}$ primeiro deles foi o Teste de Atitudes Alimentares (EAT26), o qual é especialmente utilizado para avaliar padróes alimentares e indicar uma tendência para os sintomas de anorexia nervosa. Este foi traduzido e validado para a população brasileira (alfa de Cronbach de 0,82 ). Além disso, é um instrumento identificado em revisōes sistemáticas e metanálises sobre transtornos alimentares e dança ${ }^{6,17}$. O questionário é composto por 26 questóes agrupadas em diferentes aspectos de comportamento alimentar (escala de dieta, escala de bulimia, preocupaçóes com os alimentos e escala de controle oral). As questóes do EAT-26 apresentam seis opções de resposta em escala tipo Likert (nunca, quase nunca, poucas vezes, as vezes, muitas vezes e sempre) que pontuam de zero a três. Desta forma, as respostas nunca, quase nunca e poucas vezes somam zero ponto, e as respostas as vezes, muitas vezes e sempre pontuam 1, 2 e 3 pontos, respectivamente. $\mathrm{Na}$ questão 25 , a pontuação é conferida de forma inversa às demais, somando-se zero ponto para as alternativas às vezes, muitas vezes e sempre, e 1, 2 e 3 pontos para as alternativas poucas vezes, quase nunca e nunca, respectivamente. A pontuação final do questionário pode variar de $0 \mathrm{a} 78$ pontos e o ponto de corte estabelecido pelos autores da escala original é $21^{18}$, sendo que indivíduos que somarem 21 pontos ou mais apresentam comportamento alimentar de risco ao desenvolvimento de transtornos alimentares. Assim, o resultado do EAT-26 foi classificado em duas categorias: presença de sintomas (EAT-26 $\geq 21$ ) e ausência de sintomas (EAT-26 $<21)^{18}$.

O segundo instrumento utilizado foi o Bulimic Inventory Test Edinburgh (BITE), este possui sensibilidade perfeita $(1,00)$ e alta especificidade $(0,97-0,98)$ e tem sido utilizado para detectar e avaliar a bulimia nervosa. Este instrumento foi traduzido para o português por Cordas e HochgraF ${ }^{19}$. O questionário é composto por 33 questóes e duas escalas ${ }^{17}$. A primeira escala "sintomática" possibilita uma classificação do grau dos sintomas bulímicos presentes, com no máximo trinta pontos: "compulsão e grande probabilidade de diagnóstico de bulimia" (pontuação $\geq 20$ pontos), "grupo subclínico" (15 a 19 pontos), "padrão alimentar incomum" (10 a 14 pontos) e "ausência de comportamentos típicos da bulimia” ( 0 a 9 pontos). A segunda escala, de "gravidade", fornece um índice baseado na frequência dos comportamentos compulsivos e purgativos. Os resultados podem ser "sem significância" (total $\leq 4$ pontos), "clinicamente significante" (5 a 9 pontos) ou "indícios de alta gravidade" ( $\geq 10$ pontos). Para fins estatísticos, no instrumento BITE, os participantes foram classificados na variável "na BITE sintomas" como com presença (compulsão e grande probabilidade de diagnóstico de bulimia + grupo subclínico + padrão alimentar incomum ou ausência de sintomas bulímicos) ou ausência (ausência de comportamentos típicos da bulimia) de sintomas bulímicos. Na variável "BITE gravidade", os participantes foram classificados como com presença (clinicamente significante + indícios de gravidade) ou ausência (sem significância) de sintomas bulímicos.

A análise estatística foi realizada por meio do pacote estatistico The Statistical Package for the Social Science (SPSS) - versão 20.0. Fez-se uso da estatística descritiva, mediante distribuição de frequências (absoluta e relativa). Ao verificar que a variável dependente (ansiedade traço) apresentou uma prevalência elevada, utilizou-se a regressão de Poisson com ajuste robusto para variância para estimar as associações entre este desfecho e os sintomas de transtornos alimentares (anorexia e bulimia nervosas), estimando-se razóes de prevalência (RP) e os intervalos de confiança de 95\% (IC95\%). O modelo foi ajustado pelos sintomas de transtornos alimentares, grau de escolaridade, nível econômico e modalidade da dança. $\mathrm{O}$ nível de significância adotado foi de $5 \%(\mathrm{p}<0,05)$.

\section{Resultados}

A TABELA 1 apresenta as características referentes às variáveis sociodemográficas e os sintomas de transtornos alimentares de acordo com a estratificação da ansiedade traço. Observa-se associação entre 
$\mathrm{n}(\%)$ : frequência absoluta (frequência relativa); BITE: indícios de sintomas de bulimia nervosa; EAT: indícios de sintomas de anorexia nervosa; $\mathrm{V}=\mathrm{V}$ de Cramer. ansiedade traço com os sintomas de transtornos alimentares (indícios de sintomas de anorexia e bulimia nervosas). A prevalência de ansiedade traço foi de $82,8 \%$.

TABELA 1 - Características gerais dos bailarinos participantes do Festival de dança de Joinville

\begin{tabular}{|c|c|c|c|c|}
\hline \multirow{3}{*}{ Variáveis } & \multicolumn{2}{|c|}{ Ansiedade traço } & \multirow{3}{*}{ p-valor } & \multirow{3}{*}{ V } \\
\hline & Baixa & Alta & & \\
\hline & n (\%) & n (\%) & & \\
\hline Total & $27(17,2)$ & $130(82,8)$ & & \\
\hline Grau de escolaridade & & & 0,863 & 0,056 \\
\hline Educação básica & $15(55,6)$ & $74(57,4)$ & & \\
\hline Ensino superior & $12(44,4)$ & $56(42,6)$ & & \\
\hline Nível econômico & & & 0,738 & 0,087 \\
\hline Baixo & $6(22,2)$ & $38(29,4)$ & & \\
\hline Médio & $13(48,1)$ & $58(45,0)$ & & \\
\hline Alto & $8(29,6)$ & $34(25,6)$ & & \\
\hline Modalidade dança & & & 0,240 & 0,206 \\
\hline Ballet Clássico & $6(22,2)$ & $41(31,5)$ & & \\
\hline Dança Contemporânea & $2(7,4)$ & $20(15,4)$ & & \\
\hline Danças Urbanas & $19(70,4)$ & $69(53,1)$ & & \\
\hline BITE_Sintomas & & & 0,001 & 0,393 \\
\hline Ausente & $24(91,8)$ & $82(63,6)$ & & \\
\hline Presente & $3(7,2)$ & $48(36,4)$ & & \\
\hline BITE_Gravidade & & & 0,002 & 0,298 \\
\hline Ausente & $25(92,6)$ & $81(62,3)$ & & \\
\hline Presente & $2(7,4)$ & $49(37,7)$ & & \\
\hline EAT & & & 0,027 & 0,205 \\
\hline Ausente & $24(91,8)$ & $96(75,6)$ & & \\
\hline Presente & $3(7,2)$ & $34(24,4)$ & & \\
\hline
\end{tabular}

A partir dos resultados apresentados na TABELA2, é possível observar, na análise bruta associação da bulimia nervosa (sintomas e gravidade) e anorexia com a ansiedade traço. Quando a análise foi ajustada pelos sintomas de transtornos alimentares, nível econômico, grau de escolaridade e modalidade da dança, observou-se que os bailarinos com tendência a apresentarem sintomas de bulimia nervosa tiveram $1,15(\mathrm{IC} 95 \%=1,05-1,27)$ vezes mais probabilidade de apresentar ansiedade elevada. Além disso, os bailarinos com tendência a apresentarem gravidade de bulimia nervosa apresentaram probabilidade de 1,13 (IC95\% = 1,01-1,28) de ter ansiedade traço elevada.

TABELA 2 - Associação entre ansiedade traço, bulimia e anorexia nervosa em bailarinos

\begin{tabular}{lccc}
\hline \multicolumn{1}{c}{ Variáveis } & Prevalência (\%) & RP (IC95\%) & RP (IC95\%)** \\
\hline BITE_Sintomas & & 1 & 1 \\
Ausente & 69,1 & $1,23(1,12-1,34)$ & $1,15(1,05-1,27)$ \\
Presente & 30,9 & & 1 \\
BITE_Gravidade & & 1 & $1,13(1,01-1,28)$ \\
Ausente & 69,0 & $1,22(1,11-1,34)$ & \\
Presente & 31,0 &
\end{tabular}

Continua 
Continuação

TABELA 2 - Associação entre ansiedade traço, bulimia e anorexia nervosa em bailarinos

\begin{tabular}{lccc}
\hline \multicolumn{1}{r}{ Variáveis } & Prevalência (\%) & RP (IC95\%) & RP (IC95\%)** \\
\hline EAT & & & 1 \\
Ausente & 78,8 & 1 & $1,04(0,91-1,19)$ \\
Presente & 21,2 & $1,18(1,07-1,30)$ & \\
\hline
\end{tabular}

Referente aos resultados aqui apresentados nota-se uma alta prevalência de ansiedade traço e uma associação desta variável com os sintomas de transtornos alimentares em bailarinos tanto para indícios de comportamento em anorexia nervosa quanto em bulimia nervosa.

\section{Discussão}

O objetivo geral do estudo foi analisar a associação $\mathrm{da}$ ansiedade traço com sintomas de transtornos alimentares controlados por nível socioeconômico, grau de escolaridade e modalidade em bailarinos do $31^{\circ}$ Festival de Dança de Joinville na cidade de Joinville/Santa Catarina (SC). Os resultados do presente estudo apontam elevada prevalência $(82 \%)$ de ansiedade traço, considerada maior do que a encontrada em estudo com faixa etária semelhante $(20,9 \%)^{20}$, sendo que no presente estudo a idade foi de $21 \pm 5$ anos.

Bailarinos, assim como atletas em competição, veem as apresentaçóes como uma forma de demonstrar competência frente as suas habilidades, podendo vivenciar no decorrer desta, sentimentos positivos ou negativos ligados à satisfação e/ou frustação ${ }^{3}$. Por vezes os bailarinos entram no palco ansiosos, dificultando a execução de movimentos durante a coreografia e, por conseguinte, aumentando a pressão sobre o desempenho ${ }^{4}$. Apesar disto, ainda não há um consenso que determine que a ansiedade e desempenho são fatores que se relacionam de forma inversamente proporcional ${ }^{21}$.

As evidências do presente estudo apontam associação entre ansiedade traço e uma tendência a apresentar sintomas de transtornos alimentares, principalmente no que tange a presença de sintomas na escala sintomática e na escala de gravidade de bulimia nervosa, e na presença de sintomas de anorexia nervosa. Estes achados corroboram os resultados de Fragkos e Frangos ${ }^{22}$, embora com uma amostra de não bailarinos, mas que exemplifica que os fatores psicológicos, como a ansiedade, são mais expressivos para estudantes com transtornos alimentares.

GUIMARÁEs et al. ${ }^{23}$ apontam a prática do balé como fator primordial no desencadeamento de transtornos da alimentação, salientando novamente que na prática existe uma grande preocupação com a estética, assim como achados na revisão sistemática de transtornos alimentares em bailarinos de Arcelus et al. ${ }^{6}$ que aponta que bailarinos tem três vezes mais chance de desenvolver transtornos alimentares que indivíduos que não dançam. Em estudo realizado com bailarinas de balé, encontrou-se níveis elevados de ansiedade antes da competição ${ }^{4}$. Considerando a competição esportiva um ambiente que favorece sintomas de ansiedade; a dança por encontrarse em situações de competição também pode ser considerada uma influenciadora de sintomas de ansiedade, e por consequência, bailarinos mais neuróticos, com pior imagem corporal e mais ansiosos, tem uma maior tendência a desenvolver transtornos alimentares ${ }^{7}$.

Os resultados encontrados no presente estudo podem ser explicados pelo fato da pesquisa ter sido conduzida em bailarinos durante uma das maiores competiçóes de dança do mundo, uma vez que ambiente competitivo eleva os níveis de ansiedade de bailarinos ${ }^{4}$.

Ainda, no presente estudo, observou-se que os bailarinos com tendência a apresentarem gravidade e sintomas de bulimia nervosa apresentam maior probabilidade de ter ansiedade traço elevada, independentemente do nível econômico, escolaridade ou modalidade de dança. Sabendo-se que na modalidade da dança o baixo peso corporal e a estética são supervalorizados, estes fatores podem estar atrelados ao aumento dos sintomas de transtornos alimentares, independente das covariáveis apresentadas ${ }^{24}$. O estudo de BAR et al., ${ }^{25}$ demonstra esta questão, quando aponta que bailarinos que receberam acompanhamento psicológico em relaçáo à ansiedade e imagem corporal, apresentaram menor
(\%): frequência relativa; RP: razão de prevalência; IC95\%: intervalo de confiança de $95 \%$.

BITE: indícios de sintomas de bulimia nervosa; EAT: indícios de sintomas de anorexia nervosa. ${ }^{* *}$ Ajustada pelos sintomas de transtornos alimentares, nível econômico, grau de escolaridade e modalidade da dança. 
presença de sintomas de transtornos alimentares. Demonstrando a importância destas intervençôes nas escolas e academias que atendem estes bailarinos fortalecendo o lado psicológico dos bailarinos, por vezes esquecido quando no treinamento o enfoque é o físico com a técnica perfeita.

As limitaçóes deste estudo podem estar associadas à coleta de dados, pelo fato de ocorrerem paralelas as apresentaçóes dos grupos no Festival de Dança de Joinville, os bailarinos encontravam dificuldade para realizar uma pausa nas atividades para preenchimento do questionário. Ainda contribuindo para este, o fato de as atividades ocorrerem em locais diferentes, deixando os grupos dispersos no local, com integrantes em atividades diferentes, cursos, palestras, ensaios de palco, em alojamentos, na feira da sapatilha. Outro fator limitante está relacionado ao número de bailarinos por modalidade, foram incluídos no estudo apenas bailarinos inscritos na modalidade conjunto, considera-se, no entanto, que a organização do festival optou por incluir trios na categoria conjuntos, assim aumentando a disparidade de componentes em cada modalidade. Também se ressalta que os conjuntos de danças urbanas possuíam mais integrantes, quando comparados aos conjuntos de dança contemporânea e ballet clássico, pela distinção da modalidade em si.

A partir dos achados encontrados no presente estudo, conclui-se que a ansiedade traço foi fortemente associada aos transtornos alimentares de bailarinos durante o Festival de Dança de Joinville. Dessa maneira, torna-se essencial a produção de mais pesquisas, que relacionem a ansiedade e os sintomas de transtornos alimentares na comunidade da dança, a fim de que os bailarinos possam aprender a conviver com a ansiedade durantes ensaios, competiçóes e/ou festivais e desta maneira não influencie negativamente no desempenho dos mesmos, tanto na vida profissional como pessoal.

\section{Abstract \\ Association of Anxiety dash with Eating Disorders symptoms in Dancers}

Dancers are constantly surrounded by feelings that can generate anxiety and reinforce the desire for thin body, leaving these sometimes more likely to eating disorders. Thus, this study aimed to analyse the association between trait anxiety with symptoms of eating disorders controlled by social economic level, schooling and modality in dancers from 31 Joinville Dance Festival in Joinville/Santa Catarina (SC). The study included 156 dancers from Dance Festival of Joinville - Santa Catarina, which answered a self-administered questionnaire containing sociodemographic, anthropometric measures, Inventory State-Trait Anxiety (STAI), the Eating Attitudes Test (EAT 26) and Bulimic Inventory Test Edinburgh (BITE). We observed a high prevalence of trait anxiety (82\%). There was an association between symptoms of bulimia nervosa with trait anxiety, indicating the dancers with symptoms of bulimia were $1.15(95 \% \mathrm{IC}=1.05-1.27)$ more likely to have higher anxiety and those dancers with severe symptom of bulimia had a higher probability of $1.13(95 \% \mathrm{Cl}=1.01-1.28)$ to have high trait anxiety. We found high prevalence and strong association of trait anxiety with eating disorders in dancers during the Joinville Dance Festival.

\section{KeYwords: Anxiety; Bulimia Nervosa; Anorexia Nervosa; Dancing.}

\section{Referência}

1. Nanni D. O ensino da dança na estruturação/expansão da consciência corporal e da autoestima do educando. Fitness Perform J. 2005;4(1):45-57.

2. Souza FNG, Mariani ME, Samulski DM. Análise do nível de estresse e da ansiedade embailarinos e bailarinas profissionais na pré-estreia de um espetáculo de dança. Rev Online Unileste [Internet]. 2004 [citado 30 jul 2019];1(1). Disponível em: https://www.unileste.edu.br/revistaonline/volumes/01/downloads/artigo_19.doc.

3. Constantino ACS, Prado WL, Lofrano-Prado MC. Ansiedade em bailarinos profissionais nas apresentaçóes de dança. Conexôes. 2010;8(3):146-55. 
4. Leite GSF, Mello MT, Antunes HKM. Competição na dança clássica: um fator ansiogênico negativo? Rev Bras Educ Fís Esporte. 2016;30(3):793-803.

5. Goodwin H, Arcelus J, Marshall S, Wicks S, Meyer C. Critical comments concerning shape and weight: associations with eating psychopathology among full-time dance students. Eat Weight Disord. 2014;19(1):115-8. Epub 2013 Oct 6.

6. Arcelus J, Witcomb GL, Mitchell A. Prevalence of Eating Disorders amongst Dancers: a systemic review and meta-analysis. Eur Eat Disorders Rev. 2014;22(2):92-101.

7. Liu C, Tseng MM, Chang C, Fang D, Lee M. Comorbid psychiatric diagnosis and psychological correlates of eating disorders in dance students. J Formos Med Assoc. 2016;115(2):113-20.

8. Monteiro MF, Correa MM. Transtornos alimentares em bailarinas clássicas adolescentes. Rev Bras Promoç Saúde. 2013;26(3):396-403.

9. Ribeiro LG, Veiga GV. Risk behaviors for eating disorders in brazilian dancers. Int J Sports Med. 2010;31(4):283-8.

10. Reis NM, Pelegrini A, Boing L, Monte FCSG, Simas JPN, Guimaraes ACA. Imagem corporal, estado nutricional e sintomas de transtornos alimentares em bailarinos. Rev Bras Ativ Fís Saúde. 2013;18(6):647-57.

11. Guinness World Records 2005. Rio de Janeiro: HarperCollins Brasil; 2005.

12. Associação Brasileira de Empresas de Pesquisa. Critério de classificação econômica Brasil. São Paulo: Abep; 2012.

13. Coqueiro RS, Borges LJ, Araújo VC, Pelegrini A, Barbosa AR. Medidas auto-referidas são validas para a avaliação do estado nutricional na populaçáo brasileira? Rev Bras Cineantropom Desempenho Hum. 2009;11(1):113-9.

14. Silveira EA, Araújo CL, Gigante DP, Barros AJD, Lima MS. Validação do peso e altura referidos para o diagnóstico do estado nutricional em uma população de adultos no Sul do Brasil. Cad Saúde Pública. 2005;21(1):235-45.

15. Spielberger CD, Buela-Casal G, Agudelo D, Carretero-Dios H, Santolaya F. Analysis of convergent and discriminant validity of the Spanish experimental version of the State-Trait Depression Questionnaire (ST-DEP). Actas Esp Psiquiatr. 2005;33(6):374-82.

16. Gorenstein C, Andrade L. Validation of a Portuguese version of the Beck Depression Inventory and the State-Trait Anxiety Inventory in Brazilian subjects. Braz J Med Biol Res. 1996;29(4):453-7.

17. Bigheti F, Santos CB, Santos JE, Ribeiro RPP. Tradução e avaliação do Eating Attitudes Test em adolescentes do sexo feminino de Ribeirão Preto, São Paulo. J Bras Psiquiatr. 2004;53(6):339-46.

18. Garner DM, Olmsted MP, Bohr Y, Garfinkel PE. The eating attitudes test: psychometric features and clinical correlates. Psychol Med. 1982;12(4):871-8.

19. Cordas TA, Hochgraf PB. O "BITE”: instrumento para avaliação da bulimia nervosa-versão para o português. J Bras Psiquiatr. 1993;3(42):141-44.

20. Mondin TC, Konradt CE, Cardoso TA, et al. Anxiety disorders in young people: a population-based study. Rev Bras Psiquiatr. 2013;35(4):347-52.

21. Karino CA, Laros JA. Ansiedade em situaçóes de prova: evidências de validade de duas escalas. Psico-USF. 2014;19(1):23-36.

22. Fragkos KC, Frangos CC. Assessing Eating Disorder Risk: the pivotal role of achievement anxiety, depression and female gender in non-clinical samples. Nutrients. 2013;5(3):811-28.

23. Guimarães AD, Machado SP, Franca AKTC, Calado IL. Eating disorders and dissatisfaction with body image in ballet dancers. Rev Bras Med Esporte. 2014;20(4):267-71.

24. Santos ARM, Lofrano-Prado MC, Moura PV, Silva EAPC, Leão ACC, Freitas CMSM. Ansiedade pré-competitiva em jovens atletas de nado sincronizado: uma análise a luz dos aspectos emocionais. Rev Educ Fis UEM. 2013;24(2):207-14.

25. Bar RJ, Cassin SE, Dionne MM. The long-term impact of an eating disorder prevention program for professional ballet school students: a 15-year follow-up study. Eat Disord. 2017;25(5):375-87.

\begin{tabular}{|c|c|}
\hline \multirow{7}{*}{$\begin{array}{r}\text { ENDEREÇO } \\
\text { Nycolle Martins Reis } \\
\text { Rua Tereza Lopes, } 734 \text { - Campeche }-88066-065 \\
\text { Florianópolis - SC - BRASIL } \\
\text { e-mail: nycolle_mr@hotmail.com; } \\
\text { nymreis@gmail.com }\end{array}$} & Recebido para publicação: 27/07/2015 \\
\hline & 1a. revisão: 21/06/2016 \\
\hline & 2a. revisão: 03/10/2016 \\
\hline & 3a. revisão: 28/11/2016 \\
\hline & 4a. revisão: 06/07/2018 \\
\hline & 5a. revisão: 30/o8/2018 \\
\hline & Aceito: $12 / 10 / 2018$ \\
\hline
\end{tabular}

